\title{
Teaching Notes on Subspace Method in Signal Processing
}

\author{
Qun Wan' Ji Hao Yin ${ }^{1}$ \\ ${ }^{1}$ Dept of EE, University of Electronic Science and Technology of China
}

\begin{abstract}
During the teaching of subspace method in signal processing, many students are confused about the signal models, which are dependent on different practical applications. In this paper, we introduce a simple example to reveal the basic idea of subspace method. The students are only required to have prior knowledge on definition of Euclid distance, cosine formula of triangle and eigenvalue decomposition.
\end{abstract}

Keywords: teaching, subspace method, signal processing

\section{Introduction}

During the past two decades, subspace method has been the subject of extensive research for its potential applications in science and technology, especially in radar and acoustic signal processing, wireless communication, geophysical exploration, image enhancement and image recognition $^{[1]}$.

Subspace method has played a fundamental role in the array processing applications such as position location and tracking systems. Early direction finding methods incorporate beamforming techniques $^{[2]}$ to electronically steer the array in one direction at a time and measure the corresponding output power. The major drawback of this approach is its low resolution, that is, the ability to resolve two closely spaced sources. Other direction finding approaches include Maximum Likelihood(ML) techniques ${ }^{[3]}$ and eigenstructure methods such as MUSIC (MUltiple SIgnal Classificatin) $^{[4]}$ and ESPRIT $^{[5]}$. These algorithms are so-called subspace-based high-resolution direction finding algorithms and have attracted significant attention and research interest.

However, during the teaching of subspace method in signal processing, many students are confused about the signal models, which are dependent on different practical applications. In this paper, we introduce a simple example to reveal the basic idea of subspace method. The students are only required to have prior knowledge on definition of Euclid distance, cosine formula of triangle and eigenvalue decomposition.

\section{Localization using distances}

\subsection{Problem formulation}

Consider a localization problem in a plane. The x-coordinates of $n$ points are

$$
\mathbf{x}=\left[x_{1}, x_{2}, \ldots, x_{n}\right]^{T}
$$

where []$^{T}$ denotes transposition. The corresponding y-coordinates are

$$
\mathbf{y}=\left[y_{1}, y_{2}, \ldots, y_{n}\right]^{T}
$$

The coordinate of another point is

$$
\left(x_{0}, y_{0}\right)
$$

The distances between this point and the $n$ points are 


$$
\mathbf{r}=\left[r_{1}, r_{2}, \ldots, r_{n}\right]^{T}
$$

The problem is: given $\mathbf{x}, \mathbf{y}$ and $\mathbf{r}$, how to determine $\left(x_{0}, y_{0}\right)$.

\subsection{Conventional method}

The conventional solution to the above mentioned problem is to finding the cross point of $n$ circles associated with $n$ distances. It is a nonlinear problem without closed-form solution.

\subsection{Subspace method}

Let $^{[6]}$

$$
\mathbf{A}=\left[\begin{array}{ll}
\mathbf{x}-x_{0} \mathbf{e}_{\mathbf{N}} & \mathbf{y}-y_{0} \mathbf{e}_{\mathbf{N}}
\end{array}\right]
$$

we have

$$
\begin{aligned}
& \mathbf{Q}=2 \mathbf{A} \mathbf{A}^{T} \\
& =\left[\begin{array}{cccc}
2 r_{1}^{2} & r_{1}^{2}+r_{2}^{2}-g_{12}^{2} & \cdots & r_{1}^{2}+r_{N}^{2}-g_{1 N}^{2} \\
r_{2}^{2}+r_{1}^{2}-g_{21}^{2} & 2 r_{2}^{2} & \cdots & r_{2}^{2}+r_{1}^{2}-g_{2 N}^{2} \\
\vdots & \vdots & \ddots & \vdots \\
r_{n}^{2}+r_{1}^{2}-g_{N 1}^{2} & r_{n}^{2}+r_{2}^{2}-g_{N 2}^{2} & \cdots & 2 r_{N}^{2}
\end{array}\right]
\end{aligned}
$$

where

$$
g_{k m}=\sqrt{\left(x_{k}-x_{m}\right)^{2}+\left(y_{k}-y_{m}\right)^{2}}
$$

The cosine formula is used to derive the above equation, i.e.,

$$
\begin{aligned}
& \left(x_{k}-x_{0}\right)\left(x_{m}-x_{0}\right)+\left(y_{k}-y_{0}\right)\left(y_{m}-y_{0}\right) \\
& =r_{k} r_{m} \cos \theta_{k m} \\
& =r_{k}^{2}+r_{k}^{2}-g_{k m}^{2}
\end{aligned}
$$

Obviously, the rank of matrix $\mathbf{Q}$ is not larger than 2 . In other words, the column vector of $\mathbf{A}$ is orthogonal to the null subspace of matrix $\mathbf{Q}$.

Let the eigenvalue decomposition of matrix $\mathbf{Q}$ be

$$
\mathbf{Q}=\left[\begin{array}{ll}
\mathbf{Q}_{1} & \mathbf{Q}_{2}
\end{array}\right]\left[\begin{array}{ll}
\boldsymbol{\Lambda} & \mathbf{0} \\
\mathbf{0} & \mathbf{0}
\end{array}\right]\left[\begin{array}{ll}
\mathbf{Q}_{1} & \mathbf{Q}_{2}
\end{array}\right]^{T}
$$

where $\mathbf{Q}_{1}$ is $n \times 2$ matrix, $\mathbf{Q}_{2}$ is $n \times(n-2)$ matrix, $\boldsymbol{\Lambda}$ is $2 \times 2$ diagonal matrix

Therefore, we have

$$
\mathbf{Q}_{2}^{T} \mathbf{A}=\mathbf{Q}_{2}^{T}\left[\mathbf{x}-x_{0} \mathbf{e}_{\mathbf{N}} \quad \mathbf{y}-y_{0} \mathbf{e}_{\mathbf{N}}\right]=0
$$

Straightforwardly, the above problem is solved in a closed-form as

$$
\begin{gathered}
x_{0}=\frac{\mathbf{e}_{n}^{T} \mathbf{Q}_{2} \mathbf{Q}_{2}^{T} \mathbf{x}}{\mathbf{e}_{n}^{T} \mathbf{Q}_{2} \mathbf{Q}_{2}^{T} \mathbf{e}_{n}} \\
y_{0}=\frac{\mathbf{e}_{n}^{T} \mathbf{Q}_{2} \mathbf{Q}_{2}^{T} \mathbf{y}}{\mathbf{e}_{n}^{T} \mathbf{Q}_{2} \mathbf{Q}_{2}^{T} \mathbf{e}_{n}}
\end{gathered}
$$

where

$$
\mathbf{e}_{n}=\left[\begin{array}{llll}
1 & 1 & \ldots & 1
\end{array}\right]^{T}
$$

\section{Conclusion}

Without introducing any complex signal models, teachers can also pass on the basic idea of subspace method to students who are only required to have prior knowledge on definition of Euclid distance, cosine formula of triangle and eigenvalue decomposition.

\section{Acknowledgement}

This paper is supported in part by This work was supported by ' 985 ' Excellent Teaching Team Support Project (Graduate student part) of University of Electronic Science and Technology of China (No. A1098522-02).

\section{References}

[1] P. Stoica and R. Moses, Introduction to Spectral Analysis, $2^{\text {nd }}$ ed. Upper Saddle River, NJ: Prentice-Hall, 2004.

[2] B. D. Van Veen and K. M. Buckley, Beamforming: A Versatile Approach to Spatial Filtering, IEEE ASSP Mag., vol. 5, no. 2, pp. 4-24, Apr. 1988. 
[3] P.Stoica and K.C. Sharman, Maximum likelihood direction-of-arrival estimation, IEEE Trans. Acous., Speech, and Signal Processing, vol. 38, no.7, pp. 1132-1143, Jul. 1990.

[4] R.0. Schmidt, Multiple emitter location and signal parameter estimation, IEEE Trans. on Antennas and Propagation, vol. AP-34, pp. 276-280, Mar. 1986.

[5] R. Roy and T. Kailath, ESPRITEstimation of Signal Parameters via Rotational Invariance Techniques,
IEEE Trans. Acoustics, Speech and Signal Processing, vol. ASSP-37, pp. 984-995, Jul.1989.

[6] Q. Wan, Y. J. Luo, W. L. Yang, J. Xu, J. Tang, Y. N. Peng, Mobile localization method based on multidimensional similarity analysis, IEEE International Conference on Acoustics, Speech, and Signal Processing (ICASSP'2005), March 19-23, 2005 Pennsylvania Convention Center/Marriott Hotel Philadelphia PA, USA: 1081-1084 